\title{
EVOLUÇÃO DA ÁREA DE GESTÃO DO DESENVOLVIMENTO DE NOVOS PRODUTOS: UMA ANÁLISE DA LITERATURA
}

Edivan Alexandre Ferreira (edivan.ferreira@usp.br) - PPGEP, Universidade de São Paulo (USP)

Mario Sergio Salerno (․ㅗsalerno@ usp.br) - PPGEP, Universidade de São Paulo (USP)

Tiago Paz Lasmar, (tplasmar@usp.br) - PPGEP, Universidade de São Paulo (USP)

Ana Paula Franco Paes Leme Barbosa (aleme@usp.br) - PPGEP, Universidade de São Paulo (USP)

\section{RESUMO}

O presente artigo tem por objetivo entender a evolução do campo de estudos no âmbito da Gestão do Desenvolvimento de Novos Produtos (GDNP). Para tanto, o artigo analisa os trabalhos publicados no Journal of Product Innovation Management (JPIM) sobre a GDNP a partir dos estudos realizados pela Product Development and Management Association (PDMA) junto às empresas. Os estudos mais sistemáticos sobre GDNP foram iniciados nos anos 1980, evoluindo ao longo dos anos em termos de nível de análise e variáveis analisadas. Foram levantadas dimensões orientadoras de novos estudos na área. O mapeamento de sete dimensões de melhores práticas que colaboram para o sucesso de novos produtos, o nível de maturidade da organização e o grau de novidade do produto desenvolvido são os principais pilares para evolução dos estudos deste objeto.

Palavras-chave: desenvolvimento de novos produtos, gestão da inovação, práticas de gestão da inovação

\section{INTRODUÇÃO}

Os estudos sobre práticas de desenvolvimento de produto têm elevado destaque na literatura, visto a importância da evolução dessa área para a manutenção da competitividade das organizações e, em última instância, das nações. A gestão do desenvolvimento de novos produtos é importante para reduzir riscos de alocar recursos em projetos com pouca viabilidade ou relativamente menos atraentes para as organizações.

O primeiro passo de uma organização que deseja melhorar o processo de desenvolvimento de produto ou serviço é compreender a influência das práticas no seu sucesso (KAHN et al. 2012). Logo, conhecer como ocorre o DNP, como está organizado, 
quais ferramentas são utilizadas, pode auxiliar na compreensão do desempenho, das dificuldades e indicação de melhores alternativas no processo. Kahn, Barczak e Moss (2006) destacam que tal compreensão deve ser contínua para que a organização se mantenha competitiva.

Melhores práticas podem ser definidas como técnicas, métodos, processos e atividades que colaboram com a eficácia de determinado objetivo em comparação com as demais práticas (KAHN et al., 2012). Assim, as organizações buscam identificar essas práticas com o objetivo de replicá-las (DOOLEY; SUBRA; ANDERSON, 2002).

No âmbito nacional, existem estudos sobre gestão da inovação em empresas estabelecidas, contudo não há aprofundamento nas questões de organização e gestão da inovação (DE NEGRI; SALERNO, 2005; SALERNO et al., 2010; BARBOSA et al., 2021; MELO et al., 2020; BAGNO; SALERNO; DA SILVA, 2017; SALERNO et al., 2015).

O presente artigo tem por objetivo entender a evolução do campo de estudos no âmbito da GDNP. Para tanto, o artigo analisa os trabalhos publicados no JPIM sobre o DNP a partir dos estudos realizados pela PDMA junto às empresas. Desse modo, este trabalho a partir da análise da evolução do campo e da referência internacional, estrutura diretrizes de pesquisas futuras para a área de GDNP no contexto brasileiro.

\section{METODOLOGIA}

Para alcançar o objetivo proposto, este trabalho pretende analisar um conjunto de artigos relacionados ao campo de desenvolvimento de novos produtos publicados nos últimos 30 anos no JPIM. Utilizou-se o processo de levantamento de artigos através da abordagem snowballing, seguindo as considerações de Wohlin (2014). Para este trabalho, o processo de snowballing se fez necessário, pois o conjunto da amostra se caracteriza como específica de um campo de estudo e de um grupo de pesquisadores.

Foram escolhidos 16 trabalhos publicados entre 1989 a 2016, contudo, apenas 14 artigos foram lidos na íntegra, pois os artigos publicados em eventos e o capítulo da primeira versão do Handbook do JPIM não foram disponibilizados.

Na Figura 1, identifica-se o processo de busca dos artigos da amostra. 
Figura 1- Processo de levantamento de dados Fonte: Desenvolvido pelos autores.

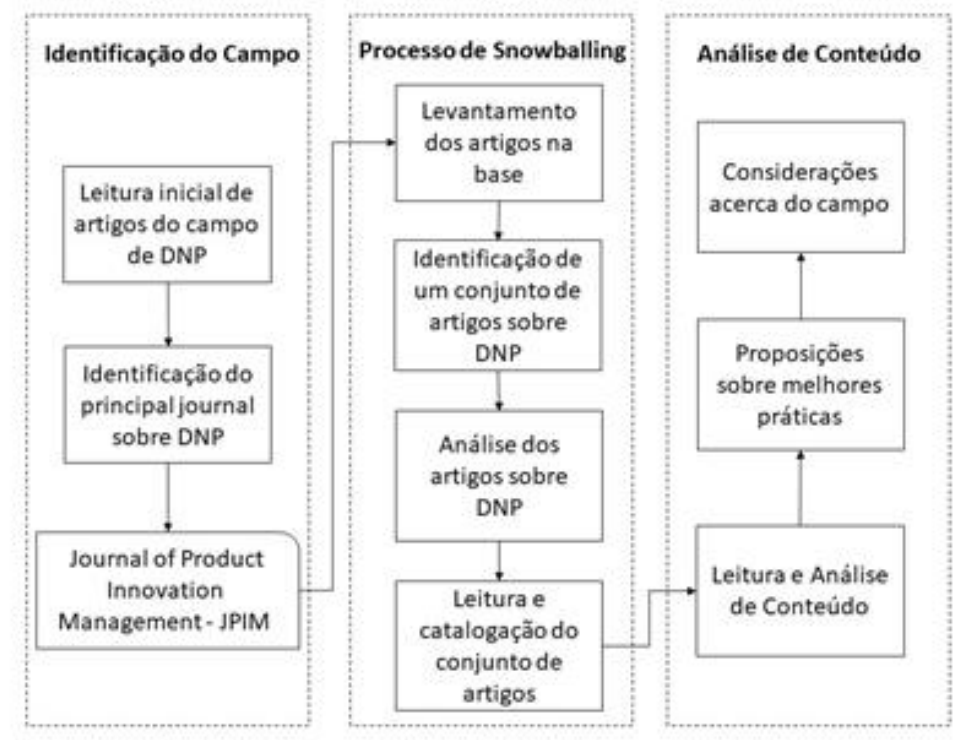

Na tabela 1, identifica-se todos os artigos levantados na pesquisa e que contribuem para o processo de identificação das melhores práticas em DNP.

Tabela 1 - Textos sobre desenvolvimento de novos produtos 


\begin{tabular}{|c|c|c|c|}
\hline$\#$ & Referência & Sintese & Tipo \\
\hline 1 & Burger (1989) & $\begin{array}{l}\text { Emergem três áreas de estudos aspectos organizacionais para o sucesso do } \\
\text { DNP, melhores práticas e análise de previsibilidade do DNP. }\end{array}$ & Artigo \\
\hline 2 & Feldman (1991) & $\begin{array}{l}\text { BP1 (1990). Perfil dos profissionais, atribuição de responsabilidade no } \\
\text { processo, setores engajados no DNP e os niveis de recompensa. }\end{array}$ & Artigo \\
\hline 3 & Page (1993) & $\begin{array}{l}\text { Mapear o processo de DNP, planos de bonificação, análise demográfica } \mathrm{e} \\
\text { métricas de medição do desempenho. }\end{array}$ & Artigo \\
\hline 4 & Griffin e Page (1993) & $\begin{array}{l}\text { Analisar cinco dimensões de sucesso e fracasso no DNP; medidas de } \\
\text { beneficios da empresa, nivel de programa, nivel de produto, desempenho } \\
\text { financeiro e aceitação do cliente. }\end{array}$ & Artigo \\
\hline 5 & Page $(1994)^{*}$ & Resultados sobre as práticas de DNP. & Evento/Conferência \\
\hline 6 & Griffin e Page (1996) & $\begin{array}{l}\text { A partir de estudos anteriores desenvolvem e testam a hipótese de que o } \\
\text { sucesso do DNP depende da estratégia de inovação da empresa. }\end{array}$ & Artigo \\
\hline 7 & Feldman (1996) & $\begin{array}{l}\text { Atualização dos resultados sobre o perfil dos profissionais publicados por } \\
\text { Feldman em } 1990 \text {. }\end{array}$ & Artigo \\
\hline 8 & Hustad (1996)* & Resultados sobre as práticas de DNP. & Capitulo de Livro \\
\hline 9 & Griffin (1997) & $\begin{array}{l}\text { BP2 (1995). Identificação de práticas e ferramentas utilizadas por } \\
\text { organizações no processo de desenvolvimento de novos produtos. }\end{array}$ & Artigo \\
\hline 10 & Markham e Griffin (1998) & $\begin{array}{l}\text { Identificar se o campeão de produto realmente influencia o desempenho do } \\
\text { DNP. }\end{array}$ & Artigo \\
\hline 11 & Adams-Bigelow (2004) & Melhores práticas de DNP. & Capitulo de Livro \\
\hline 12 & Kahn, Barczak e Moss (2006) & $\begin{array}{l}\text { Validam seis dimensões da prática de DNP: estratégia, gerenciamento de } \\
\text { portfólio, processo, pesquisa de mercado, pessoas e métricas, e } \\
\text { desempenho. }\end{array}$ & Artigo \\
\hline 13 & $\begin{array}{l}\text { Barczak, Griffin e Kahn } \\
(2009)\end{array}$ & $\begin{array}{l}\text { BP3 (2003-4) Foco em processos, ficzy front end, gestão de portfólio, } \\
\text { ferramentas de marketing e engenharia, suporte organizacional e } \\
\text { tecnológico, resultados. }\end{array}$ & Artigo \\
\hline 14 & $\begin{array}{l}\text { Kahn, Barczak, Nicholas, } \\
\text { Ledwith e Perk (2012) }\end{array}$ & $\begin{array}{l}\text { A partir de Kahn et al. (2006), redefinem as dimensões, que passam a ser } \\
\text { sete: estratégia, processo, pesquisa, clima do projeto, cultura da empresa, } \\
\text { métricas e medição de desempenho, e comercialização. }\end{array}$ & Artigo \\
\hline 15 & Markham e Lee (2013) & $\begin{array}{l}\text { BP4. Discussão do survey de } 2012 \text {, envolvendo } 24 \text { paises, com inclusão de } \\
\text { novos temas (open innovation, sustentabilidade e outros). }\end{array}$ & Artigo \\
\hline 16 & Barczak (2016) & Introdução a número especial do JPIM sobre melhores práticas em DNP. & Artigo \\
\hline
\end{tabular}

Fonte: Desenvolvido pelos autores.

\section{ANÁLISE DOS TRABALHOS}

Os surveys aplicados pela PDMA durante os 30 anos de estudos sobre DNP vêm consolidando uma base teórica rica de informações acerca das melhores práticas. Por esta razão os principais trabalhos analisados têm relação com os resultados encontrados pela pesquisa. A seguir serão discutidos os principais artigos e suas contribuições para a literatura.

\subsection{A Report on the Development of a Research Agenda for the Product Development and Management Association (1989)}

O artigo é fruto da discussão realizada no ano de 1986 com um grupo de pesquisadores associados ao PDMA, a partir de uma pesquisa bibliográfica na revista JPIM e aplicação de questionário. A proposta tinha como objetivo alinhar as metas do PDMA com os esforços de pesquisa dos pesquisadores da associação. 
Três pontos focais foram mapeados para a construção de uma agenda de pesquisa. $\mathrm{O}$ primeiro relaciona os aspectos organizacionais, buscando entender o motivo da baixa taxa de sucesso do DNP, a partir da estrutura da organização.

A segunda linha de pesquisa tinha como base os aspectos relacionados ao sucesso e fracasso do DNP. Por fim, o último ponto discutido tinha como objetivo os estudos sobre probabilidade de eventos futuros no DNP. Os autores justificaram que modelos matemáticos ou softwares eram importantes para previsão de cenários de DNP, contudo, pouca discussão sobre essa linha de pesquisa foi pesquisada ou publicada.

\subsection{A Profile of the New Product Professional (1991)}

O artigo de Feldman (1991) inicia os esforços para discussão da agenda publicada por Burger (1989). A discussão sobre o perfil dos profissionais de desenvolvimento de novos produtos está relacionada aos aspectos estruturais da organização. Assim, as informações sobre formação, nível de instrução, plano de remuneração, tempo de dedicação e amplitude de controle dos profissionais do DNP iniciam o entendimento do campo.

Os resultados indicam um número considerável de profissionais do DNP com nível de formação elevado (mestrado/doutorado). A remuneração está associada a amplitude de controle como também ao nível de formação, assim quanto maior a linha de autoridade e o nível de formação maior a remuneração. Os estudos incipientes demonstram uma visão ainda limitada sobre o campo de DNP. Objetivava-se a expansão da pesquisa permitindo que futuramente as características do perfil profissional seja uma variável no espectro do DNP.

\subsection{Assessing New Product Development Practices and Performance: establishing crucial norms (1993)}

Page (1993) investigam as melhores práticas de desenvolvimento de novos produtos. Tais achados contribuem para identificar os aspectos relacionados ao sucesso de DNP. Os principais pontos investigados foram: estrutura organizacional (foco nas divisões multifuncionais), tempo de dedicação ao DNP, características dos gerentes de projetos (campeões de produto), planos de incentivo e remuneração, processos (formais/não formais), mensuração do desempenho, problemas que dificultam o sucesso do DNP e programas de projetos. 
A pesquisa fornece um panorama das empresas em relação ao DNP, concluindo que poucas organizações têm o DNP orientado à estratégia, como também, ausência de processos formais para o DNP.

\subsection{An Interim Report on Measuring Product Development Success and Failure (1993)}

O artigo de Griffin e Page (1993) aborda a pesquisa sobre sucesso e falha no desenvolvimento de novos produtos. Baseado na agenda de Burger (1989) o artigo investiga através da literatura e na aplicação de surveys em conferências do PDMA os principais critérios que levam as organizações ao sucesso no desenvolvimento de novos produtos.

Foram identificados 75 critérios de sucesso, que por similaridade foram agrupados em categorias. Deste processo surgiram 5 principais categorias, são elas: medidas de benefícios da empresa, medidas ao nível de programa, ao nível de produto, desempenho financeiro e aceitação do cliente. Uma contribuição importante da pesquisa foi o entendimento de que as pesquisas realizadas pela academia estavam focando em macro aspectos do sucesso, como estratégia organizacional e programa de desenvolvimento, enquanto as empresas estão interessadas em descobrir os critérios ao nível de projeto. Deste modo, o trabalho propõe pesquisas futuras específicas do sucesso do projeto.

\subsection{PDMA Success Measurement Project: Recommended Measures for Product Development Success and Failure (1996)}

O artigo de Griffin e Page (1996) avalia os critérios relacionados ao sucesso e fracasso do projeto. Para os autores existem três dimensões para o sucesso do projeto: satisfação do cliente, retorno financeiro e vantagem técnica. Neste sentido os autores discutem cenários para a validação das dimensões.

O estudo identifica que muitos artigos foram publicados entre 1993 e 1994 que mapeiam os critérios de sucesso de um projeto, no total são catalogadas 75 medidas distintas. Como achados os autores identificaram que o sucesso depende da estratégia de projeto e da estratégia de negócios da empresa. Mas não há consenso entre os casos de quais medidas são realmente usadas para avaliar o sucesso do projeto.

\subsection{The Role of Salary and Incentives in the New Product Function (1996)}

Feldman (1996) atualiza e amplia as informações apresentadas por Feldman (1991) sobre o perfil dos profissionais de DNP. O estudo avalia os efeitos associados à recompensa e 
as estruturas de incentivos financeiros e obter informações acerca dos incentivos não financeiros em equipes multifuncionais de DNP.

Como resultado, a pesquisa demonstra que os incentivos financeiros aumentaram 9,6\% entre 1991 e 1996. Outros fatores já trabalhados em pesquisas anteriores foram atualizados, como o número de pessoas supervisionadas, tempo de trabalho na empresa e nível de escolaridade. A pesquisa demonstra que os incentivos relacionados ao desempenho têm maior importância à medida que o indivíduo sobe na hierarquia. Outro resultado caracterizado é a ausência de recompensas por equipe, o estudo demonstra que as recompensas são em sua grande maioria individuais.

\subsection{PDMA Research on New Product Development Practices: updating trends and} benchmarking best practices (1997)

O texto de Griffin (1997) atualiza os dados sobre as melhores práticas no desenvolvimento de novos produtos publicadas por Griffin e Page (1993). O artigo apresenta novas perspectivas, ressaltando como o sucesso depende do contexto ao qual a organização se encontra. Além disso, o autor busca separar as organizações entre as de maior e de menor desempenho, classificadas através da posição da organização no setor a qual ela pertence.

As principais análises estão relacionadas ao processo no DNP que identifica as organizações que têm processo formal/não formal, estrutura organizacional bem definida, estilo de liderança no DNP, plano de remuneração, categorias de equipe e mensuração de desempenho.

\subsection{Breakfast of champions: Associations between champions and product development environments, practices and performance (1998)}

O trabalho de Markham e Griffin (1998) discute sobre o papel dos campeões de produtos para o sucesso do projeto. A literatura sobre campeões de produtos evidencia que seu papel é fundamental para o sucesso do projeto. Desse modo, comparam o papel do campeão com o sucesso do projeto.

O estudo identifica que os campeões estão presentes em grandes e pequenas empresas, em organizações orientadas à tecnologia e organizações orientadas para o marketing. Por fim, o estudo revela que os campeões de produtos estão mais propensos a apoiar inovações radicais do que inovações incrementais (MARKHAM; GRIFFIN, 1998). 


\subsection{First Results from the 2003 Comparative Performance Assessment Study (CPAS)} (2004)

Adams-Bigelow (2004) apresenta os resultados do survey aplicado em 2003 entre os membros do PDMA. A pesquisa foi conduzida no nível da unidade de negócios e tinha como objetivo examinar as mudanças ao longo do tempo entre as pesquisas já realizadas.

Os resultados revelam que as equipes multifuncionais são predominantes entre as organizações de maior desempenho, e por fim, há um significativo uso de processos formais para a geração e seleção de ideias no front-end (ADAMS-BIGELOW, 2004).

\subsection{Perspective: establishing an NPD best practices framework (2006)}

Kahn, Barczak e Moss (2006) apresentam um framework para avaliação das melhores práticas de desenvolvimento de novos produtos, construído a partir de diversas dimensões envolvidas com o DNP. Por meio de análise bibliográfica são consolidadas 6 dimensões embasadas principalmente do trabalho de Cooper, Edgett e Kleinschmidt, (2002). As dimensões mapeadas por Kahn, Barczak e Moss (2006) são: estratégia, gestão do portfólio, processos, pesquisa de mercado, pessoas e avaliação e desempenho.

Cada dimensão está relacionada com quatro níveis, sendo o nível 1 não aconselhável e o nível 4 o desejado. Contudo, os autores trazem uma perspectiva contingencial considerando que a definição de uma estrutura ideal para o DNP pode acarretar a rigidez, sendo necessário, considerar que as dimensões podem receber graus de importância diferentes a depender do ambiente organizacional.

\subsection{Practices: results of the 2003 PDMA best practices study (2009)}

O estudo de Barczak, Griffin e Kahn (2009) analisa a pesquisa realizada em 2003 que demonstra uma evolução das dimensões tratadas pelo PDMA acerca do DNP. O artigo traz uma perspectiva histórica com análises comparativas entre os principais temas que já foram pautas em outras pesquisas como por exemplo, definição dos processos no DNP, equipes multifuncionais, plano de remuneração dos profissionais e análise de sucesso de produto.

Em resumo o artigo demonstra a evolução do campo nos quesitos de consolidação dos processos organizacionais para produtos incrementais, DNP alinhados à estratégia organizacional e importância da diversificação do portfólio de produtos. Por fim, a definição 
de ferramentas para pesquisa de mercado se mostrou importante para o sucesso do produto, por isso sua aplicação deve ser bem estruturada na organização.

\subsection{An Examination of New Product Development Best Practice (2012)}

Os estudos de Kahn, Barczak, Nicholas, Ledwith e Perks (2012) adicionam questões acerca do grau de importância das dimensões do DNP. Através da análise das dimensões, os autores constataram que um realinhamento das seis dimensões seria ideal para cobrir as principais discussões e solucionar lacunas deixadas por Kahn, Barczak e Moss (2006).

Desse modo, os autores indicam sete dimensões, são elas: estratégia, processo, pesquisa, cultura da empresa, clima do projeto, avaliação e desempenho e comercialização. Tais dimensões surgiram através da análise delph a partir de interações com especialistas da área de inovação.

\subsection{Product Development and Management Association's 2012 Comparative Performance Assessment Study (2013)}

O quarto estudo, publicado por Markham e Lee (2013), além das categorias já pesquisadas, adiciona novas perspectivas, como a cultura da inovação, sustentabilidade, propriedade intelectual e mídias sociais. Esta inserção é motivada pela importância dessas áreas nos últimos anos para o sucesso das organizações nos esforços de desenvolvimento de novos produtos.

Como no artigo de Griffin (1997), a pesquisa divide as organizações com desempenho superior das organizações de menor desempenho. Os critérios utilizados para a divisão foram: (i) as organizações consideradas mais bem-sucedidas em seu setor, (ii) a presença de um programa de DNP acima da média, (iii) lucro maior com produtos radicais em comparação com produtos incrementais. O artigo demonstra que estar entre as melhores organizações significa ter maior retorno com alguns produtos do que um portfólio repleto de produtos.

\subsection{Special Issue: Best Practices in New Product Development (2016)}

A edição especial teve como objetivo a publicação de dados relacionados ao survey de 2012. Como o volume de dados coletados foi expressivo, os editores resolveram publicar uma coletânea de artigos relacionados aos resultados da pesquisa. 
Como observado, a pesquisa realizada em 2012 foi a maior até então, o survey continha 30 páginas e 562 perguntas, e 24 países participaram da pesquisa. Os dados foram publicados em 10 artigos que abordam, seleção e geração de ideias, sustentabilidade e inovação aberta, características do sucesso dos programas de DNP e o impacto das mídias sociais para o DNP (BARCZAK, 2016).

\section{A EVOLUÇÃO DO CAMPO DE ESTUDO EM GDNP RESULTADOS}

Ao analisar o conjunto de artigos publicados pelo JPIM, identifica-se que a discussão do journal se divide em duas vertentes de pesquisa. A primeira perspectiva consiste nos surveys aplicados e analisados pelo PDMA. Ao total foram lançados quatro surveys, de 1990 a 2013. Estes evoluíram ao longo do tempo e são apresentados pelas pesquisas de Feldman (1991), Griffin (1997), Barczak, Griffin e Kahn (2009) e Markham e Lee (2013). Destaca-se a ampliação da complexidade do fenômeno de GDNP pelo crescimento substancial das variáveis analisadas pelas pesquisas investigativas do campo. As análises saíram de perspectivas pontuais, como entendimento do perfil do profissional do DNP (FELDMAN, 1991, 1996) para entender as diversas dimensões que colaboram para o bom desempenho do DNP nas empresas (GRIFFIN, 1997; MARKHAM; LEE, 2013).

Pontos importantes devem ser analisados quando se fala de melhores práticas de DNP. O avanço da competitividade no mercado colaborou para que novos aspectos fossem analisados como os esforços de inovação, a partir da sua diferenciação como radical e incremental (GRIFFIN, 2002). Ainda, o comportamento do consumidor forçou as organizações a trabalharem com ciclos de desenvolvimento de novos produtos mais curtos, buscando desenvolver com eficiência produtos com sucesso de mercado (KAHN et al., 2012). Além destas, a ascensão das mídias sociais e questões voltadas para a sustentabilidade fazem com que as organizações repensem o lançamento dos novos produtos (MARKHAM; LEE, 2013).

Além destas questões, outras variáveis são importantes, pois como discutido por Markham e Lee (2013) a função de desenvolvimento de novos produtos aumentou sua complexidade e importância para o sucesso da empresa. Assim, identificar os níveis de maturidade das dimensões do DNP (KAHN et al., 2006) e analisar as dimensões através dos níveis de inovação (MARKHAM; LEE, 2013) é fundamental para gerenciar a complexidade.

A segunda linha consiste nos esforços dos PDMA em mapear, através dos dados obtidos das pesquisas, dimensões para boas práticas no DNP. As dimensões foram publicadas 
por Kahn, Barczak e Moss (2006) e avaliadas por Kahn et al. (2012). O framework colabora para análises do DNP através de sete dimensões que apoiam o maior desempenho do DNP. A figura 2 exemplifica as dimensões propostas pela literatura.

Figura 2 - Framework para estudo do campo de DNP

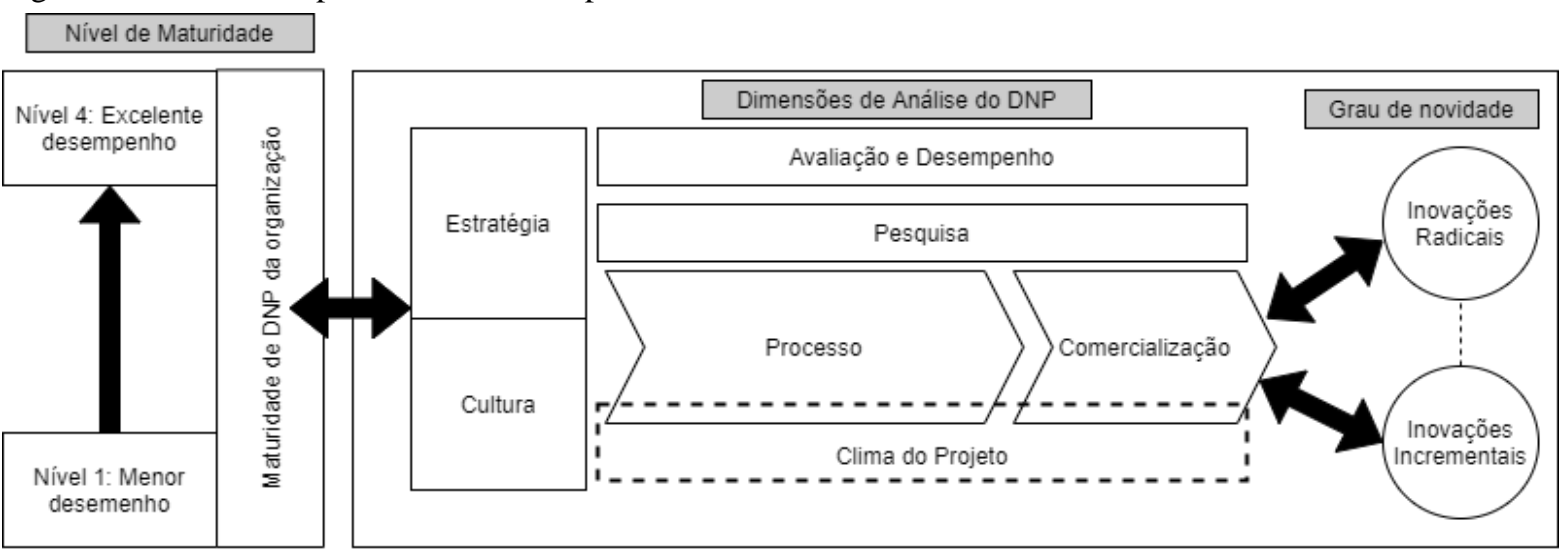

Fonte: Desenvolvido pelos autores.

A tabela 2 apresenta as dimensões e as temáticas abordadas na literatura de forma mais detalhada.

Tabela 2: Melhores práticas para o sucesso do DNP

\begin{tabular}{|c|c|}
\hline Dimensões & Critérios \\
\hline $\begin{array}{l}\text { 1 - Estratégia } \\
\text { (a) priorização de projetos; (b) objetivos de DNP } \\
\text { claros; (c) alinhamento de projetos à estratégia }\end{array}$ & $\begin{array}{l}\text { A estratégia de DNP envolve o planejamento e a definição de uma visão. Segundo Kahn et al. (2012) a } \\
\text { estratégia é voltada para pesquisa e desenvolvimento (P\&D) e esforços de desenvolvimento de novos } \\
\text { produtos. }\end{array}$ \\
\hline $\begin{array}{l}2 \text { - Processo } \\
\text { (a) processo formal de DNP; (b) documentação } \\
\text { do processo; (c) priorização de projetos }\end{array}$ & $\begin{array}{l}\text { Representação dos estágios de desenvolvimento de novos produtos. Identificação das atividades realizadas } \\
\text { que permitem a movimentação do projeto (ideação/lançamento). Inclui também, as atividades e sistemas } \\
\text { utilizados pela organização para a gestão de conhecimento. }\end{array}$ \\
\hline $\begin{array}{l}3 \text { - Pesquisa } \\
\text { (a) viabilidade tecnológica; (b) viabilidade de } \\
\text { produção; (c) pesquisa de mercado; (d) opinião } \\
\text { do cliente; (e) avaliação após o lançamento }\end{array}$ & $\begin{array}{l}\text { A pesquisa caracteriza-se como o processo de identificação da necessidade do cliente, esforços de } P \& D \text { para } \\
\text { inovar. Aplicação de metodologias e técnicas para sentir, aprender e compreender clientes, concorrentes e } \\
\text { forças macroambientais no mercado (Kahn et al., 2012). }\end{array}$ \\
\hline $\begin{array}{l}\text { 4- Cultura da Empresa } \\
\text { (a) origem das ideias (internas x externas); (b) } \\
\text { apoio da alta administração; (c) recompensas }\end{array}$ & $\begin{array}{l}\text { É um sistema de valores de gestão da organização que conduz os meios e a forma que as pessoas da } \\
\text { organização devem colaborar no desenvolvimento de novos produtos com parceiros externos (clientes e } \\
\text { parceiros). Outros fatores, como apoio da alta administração e processos de recompensas são considerados. }\end{array}$ \\
\hline \multicolumn{2}{|l|}{ 5- Clima do Projeto } \\
\hline $\begin{array}{l}\text { (a) tipo de liderança; (b) estrutura dos recursos } \\
\text { humanos; (c) gestão de equipes; (d) tempo de } \\
\text { dedicação ao DNP; (e) equipes multifuncionais }\end{array}$ & São os meios e as formas que fundamentam a integração empresa no nivel individual e de equipe. \\
\hline $\begin{array}{l}6 \text { - Avaliação e Desempenho } \\
\text { (a) relatório do desempenho do projeto; (b) } \\
\text { métricas ou critérios de avaliação; (c) processo } \\
\text { de eliminação de projetos }\end{array}$ & $\begin{array}{l}\text { A avaliação e desempenho ajuda a acompanhar durante a execução e lançamento como a organização } \\
\text { executou o projeto, além disso, a avaliar o sucesso do projeto após o lançamento. Destaca o cuidado que as } \\
\text { organizações devem ter para adaptar as métricas para atender a projetos especificos. }\end{array}$ \\
\hline \multicolumn{2}{|l|}{ 7- Comercialização } \\
\hline $\begin{array}{l}\text { (a) consolidação de canal; (b) relacionamento } \\
\text { com o cliente (CRM); (c) venda; (d) aceitação do } \\
\text { produto }\end{array}$ & $\begin{array}{l}\text { Descreve as atividades relacionadas ao marketing, lançamento e gerenciamento pós-lançamento de novos } \\
\text { produtos que estimulam a adoção do cliente e a difusão no mercado. }\end{array}$ \\
\hline
\end{tabular}

Fonte: Desenvolvido pelos autores. 
A partir da análise dos trabalhos o artigo propõe reconfigurar as dimensões com o objetivo analisá-las no contexto brasileiro, tabela 3. O processo consistiu em uma análise e refinamento das dimensões. Tais dimensões servirão de base para o andamento das pesquisas propostas neste artigo.

Tabela 3 - Síntese das dimensões do DNP

\section{Dimensões}

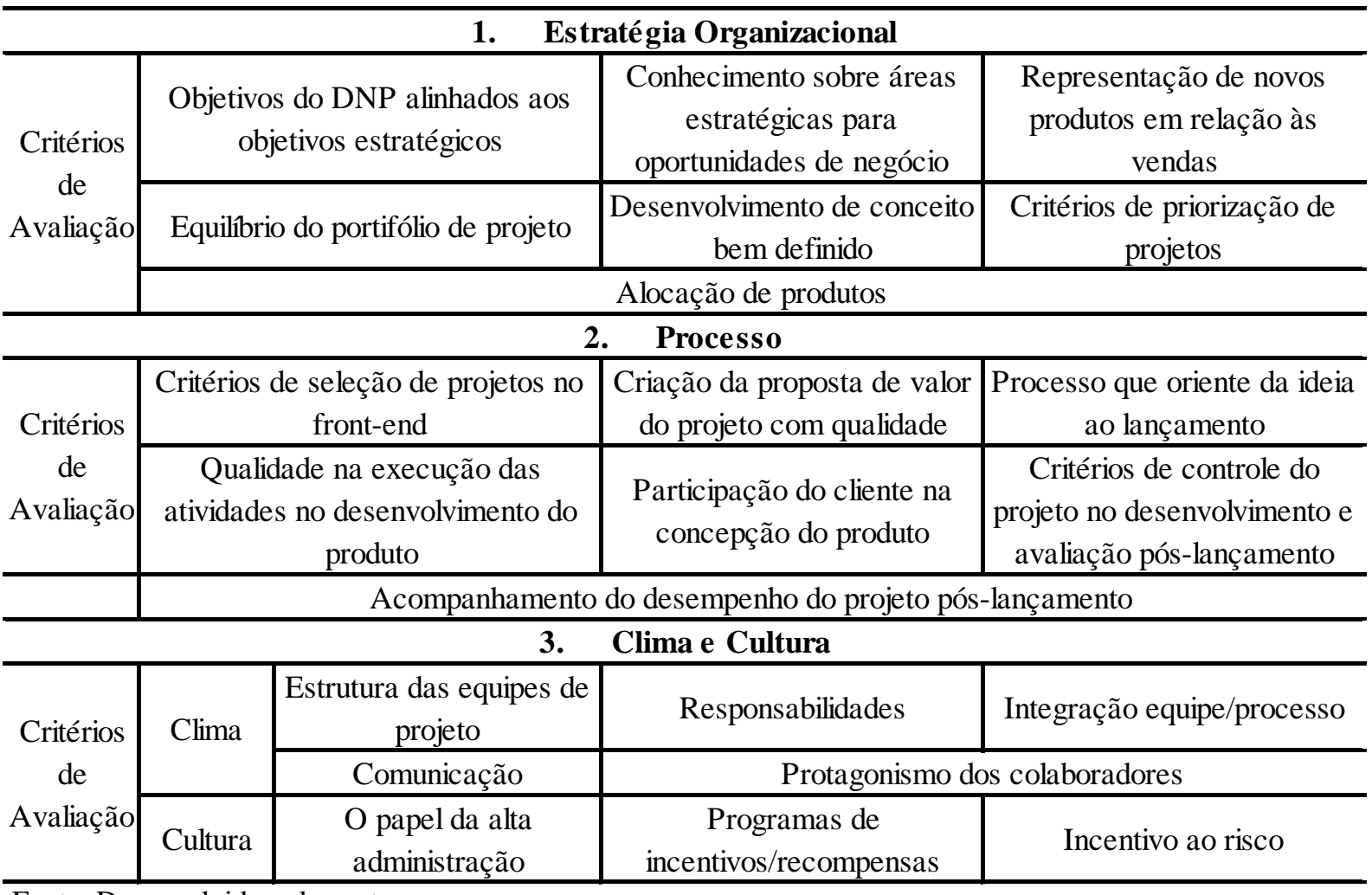

Fonte: Desenvolvido pelos autores

Estratégia consiste na tradução dos esforços organizacionais no desenvolvimento de novos produtos. Tais esforços acarretam decisões de seleção e planejamento de projetos no front-end, traduzido no desenvolvimento de conceitos, avaliação e monitoramento no desenvolvimento. Como também, a alocação de recursos nos projetos que influenciam no desempenho e no tempo de lançamento. O portfólio do projeto é agregado nesta dimensão visto os objetivos em comum, fator evidenciado e justificado por (Kahn, Barczak, Nicholas, Ledwith, \& Perks, 2012). O portfólio aborda a discussão em relação aos recursos e métodos de gerenciamento de projetos (Cooper, Edgett, \& Kleinschmidt, 2004a).

O processo é a segunda dimensão proposta nesta pesquisa. As discussões sobre processo no DNP, já foram consolidadas na literatura, sobre a ótica de modelos de processo tradicionais e sistemáticos (Cooper, 1990; Wheelwright \& Clark, 1992). Contudo, a literatura 
ainda carece de discussões sobre o front-end no que se dar em relação aos aspectos de captura de ideias e criação de propostas de valores com qualidade. Além disso, a qualidade na execução das atividades nos processos tradicionais devem ser discutidas com maior profundidade (Cooper, Edgett, \& Kleinschmidt, 2004c).

Clima e Cultura da empresa é a dimensão que discute as relações sociais da organização, debate sobre os critérios de desenvolvimento pessoal com foco nas políticas de incentivo e avaliações de desempenho e a relação da alta administração com o DNP. O clima está relacionado ao nível de projeto enquanto a cultura aborda os aspectos relacionados ao portfólio de projetos (Cooper, Edgett, \& Kleinschmidt, 2004b). Desse modo, a dimensão é subdivida em aspectos macros relacionados aos programas (portfólio) e ao nível micro, associado às discussões de projeto.

As referidas dimensões com suas respectivas melhores práticas não garantem o sucesso do projeto ou da organização, mas são ferramentas que proporcionam melhores políticas na busca por oportunidades (Cooper et al., 2004b).

\section{CONCLUSÃO}

O presente trabalho teve como objetivo elucidar a evolução do campo de estudo relacionado à GDNP. A partir de uma revisão de literatura de textos publicados no JPIM e dos estudos realizados pelo PDMA. Foram levantados pilares fundamentais para a validação das dimensões. O mapeamento de sete dimensões de melhores práticas na literatura fora adaptado, assim, as melhores práticas de cada dimensão serão avalidas em futuras pesquisas.

Como pesquisas futuras sugere-se o estudo de combinação de melhores práticas para o melhor desempenho do DNP, buscando ir além de uma associação direta entre apenas uma boa prática e o sucesso do desenvolvimento. Ainda é possível o desenvolvimento de novos estudos capazes de se fazer adaptações das principais dimensões de análises considerando as melhores práticas em diferentes contextos.

\section{AGRADECIMENTOS}

A pesquisa foi parcialmente financiada pela Fapesp - Fundação de Amparo à Pesquisa do Estado de São Paulo, processo 2015/26662-5. 


\section{REFERÊNCIAS}

ADAMS-BIGELOW, M. First Results from the 2003 Comparative Performance Assessment Study (CPAS). The PDMA Handbook of New Product Development, n. December, p. 546-566, 2004.

BAGNO, R. B.; SALERNO, M. S.; DA SILVA, D. O. Models with graphical representation for innovation management: a literature review. $\mathbf{R}$ and $\mathbf{D}$ Management, v. 47, n. 4, p. 637653, 2017.

BARBOSA, A. P. F. P. L. et al. Configurations of project management practices to enhance the performance of open innovation R\&D projects. International Journal of Project Management, v. 39, n. 2, p. 128-138, 2021.

BARCZAK, G. Special Issue: Best Practices in New Product Development. Journal of Product Innovation Management, v. 33, p. 2-2, 1 dez. 2016.

BARCZAK, G.; GRIFFIN, A.; KAHN, K. B. Perspective: Trends and drivers of success in NPD practices: Results of the 2003 PDMA best practices study. Journal of Product Innovation Management, v. 26, n. 1, p. 3-23, 2009.

BURGER, P. C. A Report on the development of a research agenda for the product development and management association. Journal of Product Innovation Management, v. 6, p. 51-60, 1989.

CARVALHO, M. M.; FLEURY, A.; LOPES, A. P. An overview of the literature on technology roadmapping (TRM): Contributions and trends. Technological Forecasting and Social Change, v. 80, n. 7, p. 1418-1437, 1 set. 2013.

DE NEGRI, J. A.; SALERNO, M. S. Inovações, Padrões Tecnológicos e Desempenho das Firmas Industriais Brasileiras. Brasília: [s.n.]. Disponível em: <http://www.ipea.gov.br>. Acesso em: 22 jan. 2021.

DOOLEY, K. J.; SUBRA, A.; ANDERSON, J. Adoption Rates and Patterns of Best Practices in New Product Development. International Journal of Innovation Management, v. 06, n. 01, p. 85-103, 2002.

FELDMAN, L. P. A profile of the new product professional. The Journal of Product Innovation Management, v. 8, n. 4, p. 252-266, 1991.

FELDMAN, L. P. The role of salary and incentives in the new product function. Journal of Product Innovation Management, v. 13, n. 3, p. 216-228, 1996.

GRIFFIN, A. PDMA research on new product development practices: Updating trends and benchmarking best practices. Journal of Product Innovation Management, v. 14, n. 6, p. 429-458, 1997.

GRIFFIN, A. Product development cycle time for business-to-business products. Industrial Marketing Management, v. 31, n. 4, p. 291-304, 2002.

GRIFFIN, A.; PAGE, A. L. An Interim Report on Measuring Product Development Success and Failure. Journal of Product Innovation Management, v. 10, n. 4, p. 291-308, 1993.

GRIFFIN, A.; PAGE, A. L. Abbie Griffin and Albert L. Page. Journal of Product Innovation Management, v. 13, n. 96, p. 478-496, 1996.

KAHN, K. B. et al. An examination of new product development best practice. Journal of Product Innovation Management, v. 29, n. 2, p. 180-192, 2012.

KAHN, K. B.; BARCZAK, G.; MOSS, R. Perspective: Establishing an NPD Best Practices Framework. Journal of Product Innovation Management, v. 23, n. 2, p. 106-116, 2006.

MARKHAM, S. K.; GRIFFIN, A. Breakfast of champions: Associations between champions and product development environments, practices and performanceJournal of Product Innovation Management, 1998. 
MARKHAM, S. K.; LEE, H. Product development and management association's 2012 comparative performance assessment study. Journal of Product Innovation Management, v. 30, n. 3, p. 408-429, 2013.

MELO, J. C. F. DE et al. From open innovation projects to open innovation project management capabilities: A process-based approach. International Journal of Project Management, v. 38, n. 5, p. 278-290, 2020.

PAGE, A. L. Assessing new product development practices and performance: Establishing crucial norms. The Journal of Product Innovation Management, v. 10, n. 4, p. 273-290, 1993.

SALERNO, M. S. et al. Inovação: estudos de jovens pesquisadores brasileiros. São Paulo: Editora Papagaio, 2010.

SALERNO, M. S. et al. Innovation processes: Which process for which project? Technovation, v. 35, p. 59-70, 2015.

SILVA, D. O. DA; BAGNO, R. B.; SALERNO, M. S. Modelos para a gestão da inovação, revisão e análise da literatura. Production, v. 24, n. 2, p. 477-490, 2014.

WOHLIN, C. Guidelines for Snowballing in Systematic Literature Studies and a Replication in Software Engineering. 18th International Conference on Evaluation and Assessment in Software Engineering. Anais...2014Disponível em: <http://dx.doi.org/10.1145/2601248.2601268>. Acesso em: 10 jun. 2021 